\title{
High postoperative neutrophil-to-lymphocyte ratio as a poor prognostic marker in patients with upper tract urothelial carcinoma
}

\author{
KIYOAKI NISHIHARA, SHIGETAKA SUEKANE, KOUSUKE UEDA, \\ MAKOTO NAKIRI, MITSUNORI MATSUO and TSUKASA IGAWA \\ Department of Urology, Kurume University School of Medicine, Kurume, Fukuoka 830-0011, Japan \\ Received September 14, 2018; Accepted February 20, 2019
}

DOI: $10.3892 / \mathrm{ol} .2019 .10178$

\begin{abstract}
Previously, the preoperative neutrophil-to-lymphocyte ratio (NLR) has been demonstrated to be a beneficial prognostic marker in patients with upper tract urothelial carcinoma (UTUC). However, to the best of our knowledge, the postoperative NLR has rarely been investigated. Therefore, the present study evaluated the prognostic significance of postoperative NLR in patients with UTUC. Data of patients with UTUC who underwent surgical treatment at Kurume University hospital (Kurume, Japan) between 2004 and 2015 were retrospectively reviewed. Clinicopathological characteristics were analyzed, including pre- and postoperative NLRs. Overall survival (OS) and cancer-specific survival (CSS) rates were estimated using the Kaplan-Meier method and compared with a log-rank test. Multivariate proportional Cox regression models were applied for both endpoints to identify the independent prognostic significance of NLR. The median age of the 134 enrolled patients was 70 years. The postoperative NLR was elevated in 35 patients (26.1\%). A high postoperative NLR of $\geq 2.5$ was significantly associated with a high postoperative C-reactive protein level of $\geq 0.3 \mathrm{mg} / \mathrm{dl}$, an advanced pathological $\mathrm{T}$ stage and positive lymphovascular invasion in surgical specimens $(\mathrm{P}<0.001, \mathrm{P}=0.019$ and $\mathrm{P}=0.024$, respectively). The 5-year OS rates in patients with high and low postoperative NLR were 33.7 and $70.2 \%$, respectively $(\mathrm{P}<0.001)$, and the 5-year CSS rates in patients with a high and low postoperative NLR were 33.7 and $80.7 \%$, respectively $(\mathrm{P}<0.001)$. Multivariate analysis revealed that a high postoperative NLR was an independent
\end{abstract}

Correspondence to: Dr Kiyoaki Nishihara, Department of Urology, Kurume University School of Medicine, 67 Asahi-machi, Kurume, Fukuoka 830-0011, Japan

E-mail: nishihara_kiyoaki@kurume-u.ac.jp

Abbreviations: UTUC, upper tract urothelial carcinoma; NLR, neutrophil-to-lymphocyte ratio; pT, pathological T; CRP, C-reactive protein; OS, overall survival; CSS, cancer-specific survival

Key words: neutrophil-to-lymphocyte ratio, prognostic marker, surgery, survival, upper tract urothelial carcinoma prognostic marker for OS (hazard ratio, 4.66; 95\% confidence interval, 2.11-10.00; $\mathrm{P}<0.001$ ) and CSS (hazard ratio, 10.90; 95\% confidence interval, 4.32-28.40; $\mathrm{P}<0.001$ ), and the preoperative NLR was not identified as a prognostic marker. In conclusion, a high postoperative NLR is associated with a poor prognosis in patients with UTUC. Therefore, postoperative NLR may be a potential prognostic marker in patients with UTUC undergoing nephroureterectomy.

\section{Introduction}

Upper tract urothelial carcinoma (UTUC) arises from the urothelium of the renal pelvis or ureter, and $>90 \%$ of these tumors are histopathologically categorized as urothelial carcinomas (1). UTUC is uncommon, accounting for $\sim 5-10 \%$ of all cases of urothelial cancer in the United States in $2012(2,3)$. However, in recent years, the number of patients with UTUC has been increasing in numerous countries, including Japan (4). In total, 55\% of UTUC cases are muscle invasive at the initial diagnosis, which is increased compared with that of bladder cancer $(30 \%)(5,6)$. Radical nephroureterectomy with bladder cuff excision is the standard surgical treatment for localized UTUC $(1,3,5)$. However, since postoperative local relapse or distant metastasis occurs in $24-28 \%$ of cases, UTUC is considered to exhibit a poor prognosis $(5,7)$. Therefore, the proportion of invasive muscle cancer, postoperative relapse and distant metastasis in UTUC is increased, compared with the proportion of that associated with bladder cancer. Consequently, patients with UTUC have a poor prognosis, compared with patients with bladder cancer. Additionally, compared with bladder cancer, a limited number of studies have investigated predictors for patients with UTUC $(3,5-7)$. Therefore, biomarkers are required to improve the prognosis of patinets with UTUC.

The currently recognized prognostic markers for UTUC are mostly derived from pathological features, including pathological grade, T stage, lymph node involvement, surgical margin status and lymphovascular invasion $(3,8)$. However, to the best of our knowledge, it remains unknown which prognostic markers are associated with the survival outcomes of patients with UTUC.

Previously, the neutrophil-to-lymphocyte ratio (NLR) has been reported to be a beneficial prognostic marker in 
numerous types of malignancy, including lung, renal, gastric, hepatic and colorectal cancer $(9,10)$. With respect to urothelial carcinoma, the majority of previous studies regarding bladder cancer (11-13) and UTUC $(14,15)$ have only discussed preoperative NLR. In particular, to the best of our knowledge, the postoperative NLR has rarely been investigated. Therefore, the present study investigated the clinical significance of the postoperative NLR as a prognostic marker in patients with clinically localized UTUC.

\section{Materials and methods}

Study cohort and design. The present study was approved by the Research Ethics Committee of Kurume University (Kurume, Japan). Data of 152 patients diagnosed with localized UTUC who underwent radical nephroureterectomy at Kurume University hospital (Kurume, Japan) between January 2004 and December 2015 were retrospectively reviewed. Patients were excluded according to following criteria: i) Had received neoadjuvant chemotherapy; ii) had an active infection, or iii) had been administered cortical steroids. A total of 134 patients who were observed for a minimum of 6 months were included in the analysis. A diagnosis of localized UTUC was established by computed tomography/magnetic resonance imaging, urine cytology, and cystoscopy and/or ureteroscopy. Pathological T (pT) stages were uniformly adjusted according to the 2009 Tumor-Node-Metastasis classification system (16). Tumor grade was assessed according to the 1999 World Health Organization classification (17). Postoperative surveillance was performed by physical examination, urine cytology and cystoscopy at 3-month intervals, and blood examinations and computed tomography at 6-month intervals. Metastasis and local relapse were defined as urothelial tumor relapse outside the residual urinary tract. The clinicopathological characteristics of the 134 patients enrolled in the present study, and the comparisons between the high and low NLR groups are presented in Table I. The median age of the patients at the time of surgery was 70 years (range, 64-76 years) and its incidence was $\sim 2$-fold higher in male patients compared with female patients. The median surveillance time was 40.5 months (range, 20.8-71.3 months). Clinicopathological variables affecting survival were analyzed, including age at the time of surgery, sex, tumor location, the presence of hydronephrosis, tumor diameter, pre- and postoperative NLR, postoperative C-reactive protein (CRP) level, pT stage, tumor grade, the presence of lymphovascular invasion, positive surgical margins and lymph node involvement. All pathological diagnoses were performed by a specialist pathologist at Kurume University (Kurume, Japan). All clinicopathological data were retrieved from medical records at the hospital.

$N L R$. The NLR was derived by dividing the absolute neutrophil count by the absolute lymphocyte count in peripheral blood. A high NLR ( $\geq 2.5)$ was defined according to previous studies $(12,18)$. Receiver operating characteristic (ROC) curves were plotted of the postoperative NLR for the evaluation of overall survival (OS) and cancer-specific survival (CSS) rates. The area under the curves of postoperative NLR for OS and CSS were 0.617 and 0.656 , respectively.

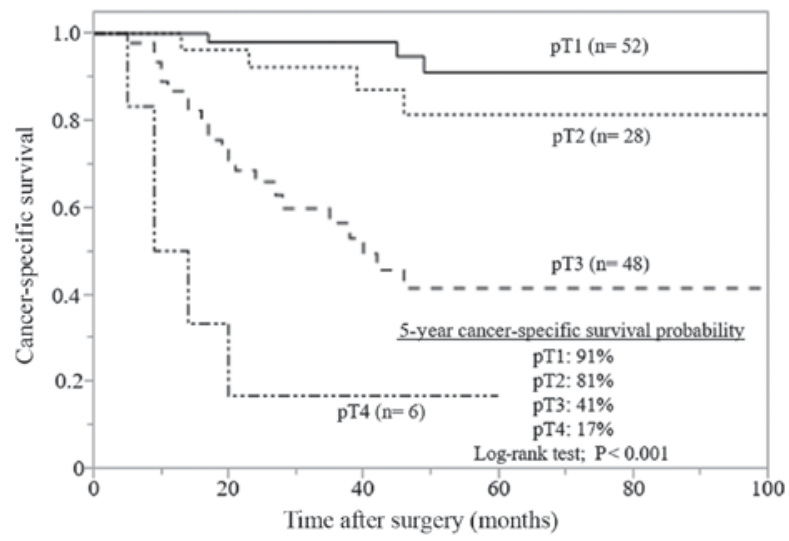

Figure 1. Kaplan-Meier curves for CSS of the 134 patients treated with radical nephroureterectomy for UTUC stratified by pT stage. CSS, cancer-specific survival; UTUC, upper tract urothelial carcinoma; pT, pathological T.

The postoperative NLR threshold was 2.67 for OS and CSS. The sensitivity of postoperative NLR for OS and CSS was 40.0 and $52.9 \%$ respectively, and the specificity was 87.6 and $89 \%$ respectively. Additionally, the NLR threshold according to the ROC curves was 2.67. In the majority of previous studies on urothelial carcinoma, the NLR threshold has been in the range 2.2-3.0 $(9,12,14,18)$. Therefore, the present study considered that 2.5 was a reasonable threshold. The preoperative NLR was measured prior to any tumor manipulation, including ureteroscopy or retrograde pyelography. The postoperative NLR was measured 1-2 months after surgery. In cases requiring adjuvant chemotherapy, the postoperative NLR was measured prior to adjuvant chemotherapy.

Statistical analysis. Mann-Whitney $\mathrm{U}$ test and $\chi^{2}$ test were used to assess differences between the high and low postoperative NLR groups. The statistical analysis data are presented as the median and interquartile range for continuous variables or the proportion of events for categorical variables. Spearman's correlation coefficient was analyzed between the preoperative NLR and postoperative NLR. Survival curves were estimated using the Kaplan-Meier method and compared with a log-rank test. Survival data were collected on December 31, 2016. Patients where contact was lost during follow-up were evaluated at the date of last contact. Patients who were alive on December 31, 2016 were assessed for OS rate. CSS rate was calculated from the date of surgery to the date of cancer-associated mortality. Patients were assessed at the date of mortality if they succumbed to mortality from other causes. OS was calculated from the date of surgery to the date of mortality from any cause. Univariate and multivariate analyses were performed using Cox proportional hazards regression models. All statistical analyses were conducted using JMP software for Windows (v.12.0.1; SAS Institute Inc., Cary, NC, USA). $\mathrm{P}<0.05$ was considered to indicate a statistically significant difference.

\section{Results}

Effect of Kaplan-Meier on $p T$ stage distribution. The $\mathrm{pT}$ stage distribution was as follows: T1 (including Ta and Tis), 38.8\%; $\mathrm{T} 2,20.9 \%$; $\mathrm{T} 3,35.8 \%$ and $\mathrm{T} 4,4.5 \%$. In total, $34 \%$ of patients 
Table I. Clinicopathological characteristics of the 134 patients with UTUC.

\begin{tabular}{|c|c|c|c|}
\hline \multirow[b]{2}{*}{ Characteristics } & \multicolumn{2}{|c|}{ Postoperative NLR } & \multirow[b]{2}{*}{ P-value } \\
\hline & $\geq 2.5(n=35)$ & $<2.5(\mathrm{n}=99)$ & \\
\hline Median age, years (IQR) & $73(66-77)$ & $70(61-76)$ & 0.142 \\
\hline \multicolumn{4}{|l|}{ Sex, n $(\%)$} \\
\hline Male & $19(54.3)$ & $69(69.7)$ & \multirow[t]{2}{*}{0.103} \\
\hline Female & $16(45.7)$ & $30(30.3)$ & \\
\hline \multicolumn{4}{|l|}{ Tumor location, n (\%) } \\
\hline Renal pelvis & $19(54.3)$ & $55(55.6)$ & \multirow[t]{2}{*}{0.312} \\
\hline Ureter & $16(45.7)$ & $44(44.4)$ & \\
\hline \multicolumn{4}{|l|}{ Hydronephrosis, n (\%) } \\
\hline Yes & $21(60.0)$ & $62(62.6)$ & \multirow[t]{2}{*}{0.784} \\
\hline No & $14(40.0)$ & $37(37.4)$ & \\
\hline Median tumor size, cm (IQR) & $3.0(1.5-3.6)$ & $2.5(1.7-3.5)$ & 0.353 \\
\hline \multicolumn{4}{|l|}{ pT stage, $\mathrm{n}(\%)(16)$} \\
\hline $\mathrm{pT} 1 / \mathrm{T} 2$ & $15(42.9)$ & $65(65.7)$ & \multirow[t]{2}{*}{$0.019^{b}$} \\
\hline $\mathrm{pT} 3 / \mathrm{T} 4$ & $20(57.1)$ & $34(34.3)$ & \\
\hline \multicolumn{4}{|l|}{ Lymph node involvement, n (\%) } \\
\hline $\mathrm{pNx} / \mathrm{N} 0$ & $28(80.0)$ & $89(89.9)$ & \multirow[t]{2}{*}{0.146} \\
\hline $\mathrm{pN} 1$ & $7(20.0)$ & $10(10.1)$ & \\
\hline \multicolumn{4}{|l|}{ Tumor grade, n (\%) } \\
\hline $\mathrm{G} 1 / \mathrm{G} 2$ & $19(54.3)$ & $70(70.7)$ & \multirow[t]{2}{*}{0.081} \\
\hline G3 & $16(45.7)$ & $29(29.3)$ & \\
\hline \multicolumn{4}{|l|}{ Lymphovascular invasion, n (\%) } \\
\hline Yes & $18(52.9)$ & $30(30.9)$ & \multirow[t]{2}{*}{$0.024^{\mathrm{b}}$} \\
\hline No & $16(47.1)$ & $67(69.1)$ & \\
\hline \multicolumn{4}{|l|}{ Surgical margins, n (\%) } \\
\hline Positive & $5(14.3)$ & $7(7.1)$ & \multirow[t]{2}{*}{0.219} \\
\hline Negative & $30(85.7)$ & $92(92.9)$ & \\
\hline \multicolumn{4}{|l|}{ Postoperative CRP level, mg/dl, n (\%) } \\
\hline$\geq 0.3$ & $12(36.4)$ & $7(7.5)$ & \multirow[t]{2}{*}{$<0.001^{\mathrm{b}}$} \\
\hline$<0.3$ & $21(63.6)$ & $86(92.5)$ & \\
\hline Median postoperative leucocytes per $\mu \mathrm{l}$ (IQR) & $5,600(4,800-7,100)$ & $5,100(4,300-6,000)$ & 0.061 \\
\hline Median postoperative neutrophils per $\mu \mathrm{l}$ (IQR) & $3,694(3,014-4,807)$ & $2,736(2,112-3,321)$ & $<0.001^{\mathrm{b}}$ \\
\hline Median postoperative lymphocytes per $\mu 1$ (IQR) & $1,222(972-1,440)$ & $1,819(1,505-2,129)$ & $<0.001^{\mathrm{b}}$ \\
\hline
\end{tabular}

had tumor grade 3. Kaplan-Meier curves for CSS stratified according to pT stage are presented in Fig. 1. Advanced pT stage ( $\geq \mathrm{T} 3)$ was associated with a significantly reduced survival, compared with early pT stage (T1 and T2; $\mathrm{P}<0.001)$.

Clinicopathological characteristics and survival analysis of postoperative NLR. The median pre- and postoperative NLRs were 2.03 (1.62-2.75) and 1.72 (1.34-2.59), respectively. The pre- and postoperative NLRs were elevated in 41 (30.6\%) and 35 patients $(26.1 \%)$, respectively (data not shown). A high postoperative NLR of $\geq 2.5$ was significantly associated with a high postoperative CRP level of $\geq 0.3 \mathrm{mg} / \mathrm{dl}(\mathrm{P}<0.001)$, an advanced pT stage $(\geq \mathrm{T} 3 ; \mathrm{P}=0.019)$ and positive lymphovascular invasion $(\mathrm{P}=0.024)$ in the surgical specimens (Table I). The median OS and CSS times for patients with a high postoperative NLR were both 35 months. The median OS and CSS times for patients with a low postoperative NLR were not reached. The 5-year OS and CSS rates in patients with a high postoperative NLR were both $33.7 \%$, and the 5-year OS and CSS rates for patients with a low postoperative NLR were 70.2 and $80.7 \%$, respectively. The Kaplan-Meier curves demonstrated that a high postoperative NLR is associated with significantly reduced OS and CSS rates, compared with a low postoperative NLR (both $\mathrm{P}<0.001$; Fig. 2). 


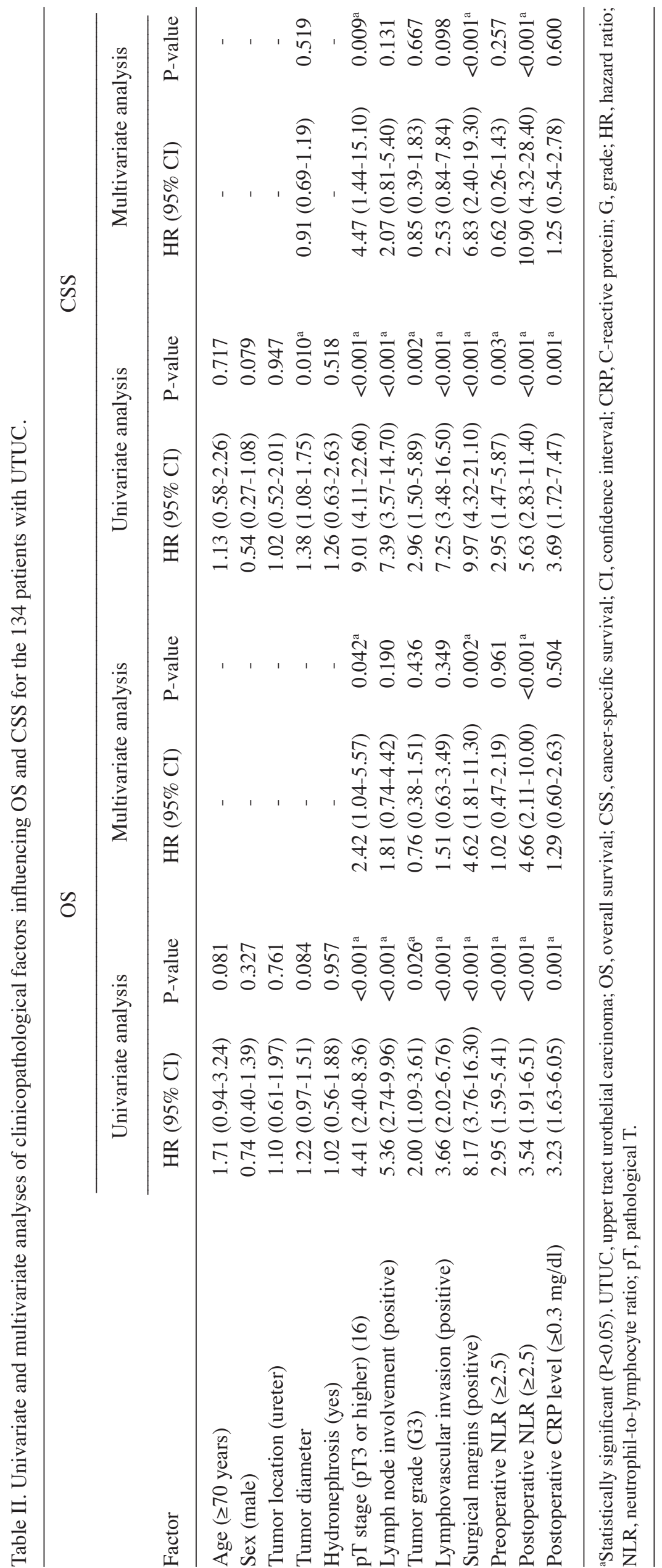




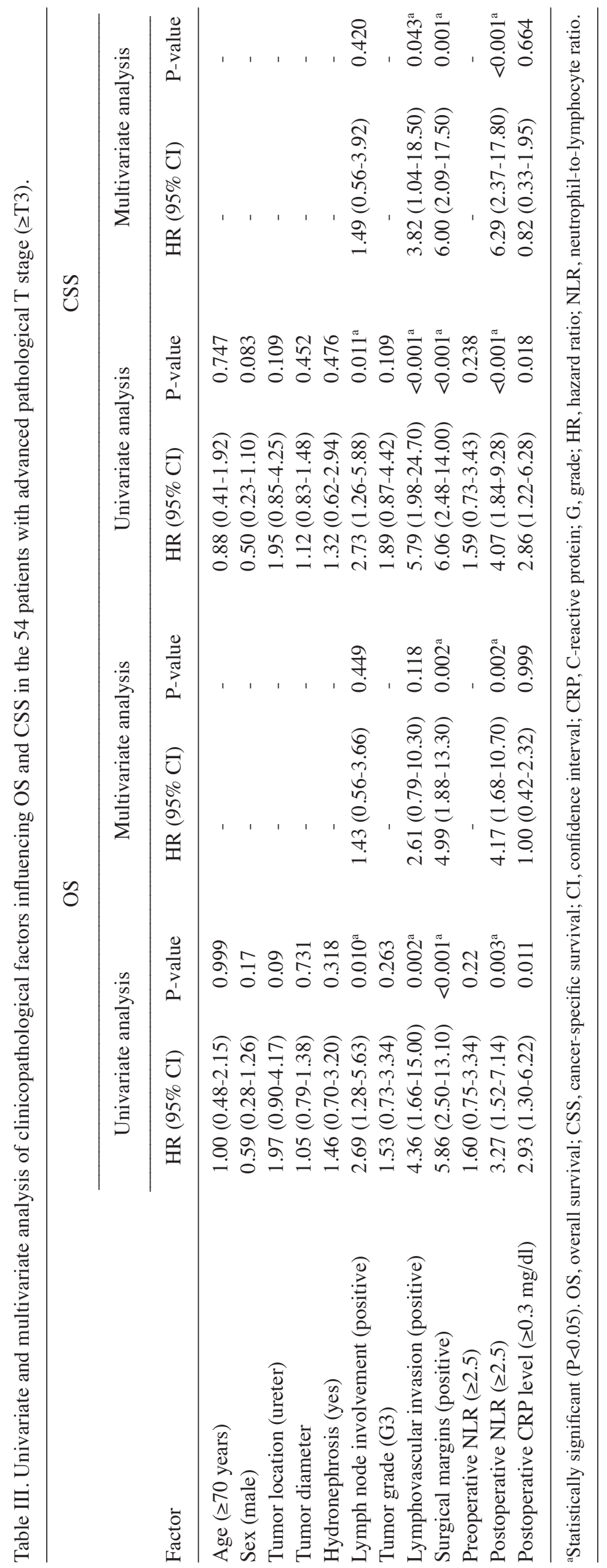



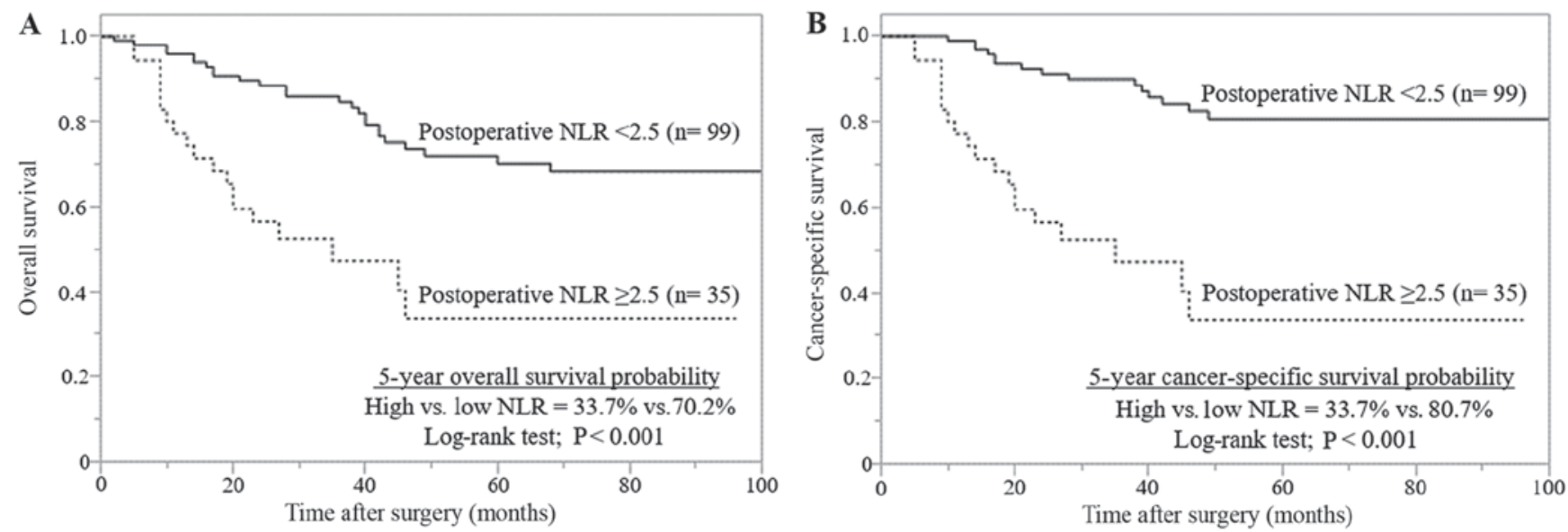

Figure 2. Survival analysis according to postoperative NLR for all patients. Kaplan-Meier curves for (A) OS and (B) CSS. NLR, neutrophil-to-lymphocyte ratio; OS, overall survival; CSS, cancer-specific survival.
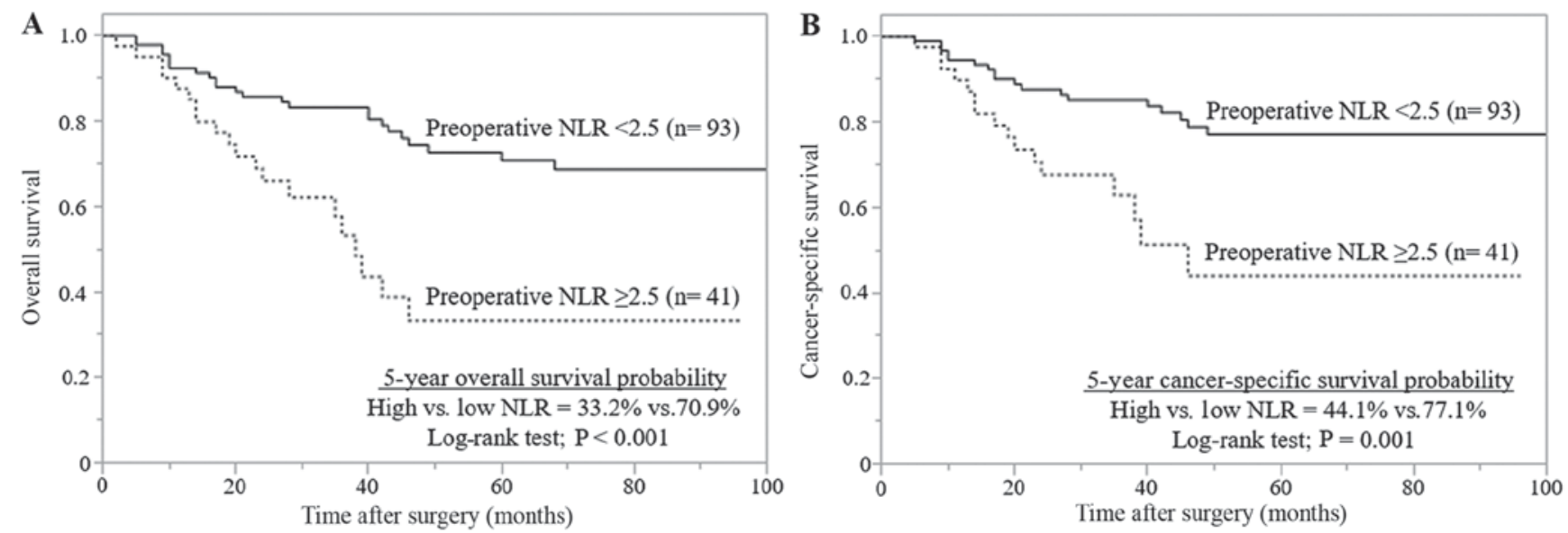

Figure 3. Survival analysis according to preoperative NLR for all patients. Kaplan-Meier curves for (A) OS and (B) CSS. NLR, neutrophil-to-lymphocyte ratio; OS, overall survival; CSS, cancer-specific survival.

Univariate and multivariate analysis. Univariate analysis revealed that advanced $\mathrm{pT}$ stage $(\geq \mathrm{T} 3)$, positive lymph node involvement, high-grade tumors, positive lymphovascular invasion, positive surgical margins, high postoperative CRP levels, and high pre- and postoperative NLRs were significantly associated with OS and CSS. Multivariate analysis identified a high postoperative NLR as an independent prognostic marker for OS and CSS (both $\mathrm{P}<0.001$ ). Additionally, advanced pT stage $(\geq \mathrm{T} 3 ; \mathrm{P}=0.042$ and $\mathrm{P}=0.009$, respectively) and positive surgical margins $(\mathrm{P}=0.002$ and $\mathrm{P}<0.001$, respectively) were identified as independent prognostic factors for OS and CSS (Table II). A high preoperative NLR was significantly associated with poor survival, compared with low preoperative NLR (Fig. 3) and the Spearman correlation coefficient demonstrated a significant correlation between the preoperative and postoperative NLR $(\mathrm{r}=0.529, \mathrm{P}<0.001$; data not shown). However, a high preoperative NLR was not a significant prognostic marker according to the multivariate analysis (Table II).

Similar results were obtained for the 54 patients (40.3\%) with advanced pT stage ( $\geq \mathrm{T} 3$ ). In total, 20/54 (37.0\%) patients had a high postoperative NLR (data not shown). In the Kaplan-Meier analysis, a high postoperative NLR of $\geq 2.5$ was significantly associated with a reduced $\mathrm{OS}(\mathrm{P}=0.001)$ and CSS
$(\mathrm{P}<0.001)$, compared with a low postoperative NLR (Fig. 4). Multivariate analysis identified high postoperative NLR as a significant independent prognostic marker for OS $(\mathrm{P}=0.002)$ and CSS $(\mathrm{P}<0.001$; Table III).

Clinicopathological characteristics and survival analysis of advanced pT stage. In total, 29/54 (53.7\%) patients with advanced pT stage ( $\geq \mathrm{T} 3)$ received adjuvant chemotherapy (Table IV). Cisplatin-based chemotherapy in various combinations was the most commonly administered therapy. A total of 19 patients (65.5\%) received cisplatin plus gemcitabine; 6 patients (20.7\%) received methotrexate, vinblastine, doxorubicin and cisplatin; 3 patients (10.3\%) received carboplatin plus gemcitabine; and 1 patient (3.5\%) received gemcitabine plus paclitaxel. The median number of chemotherapy cycles was two. Compared with those who underwent surgical treatment alone, patients who received adjuvant chemotherapy exhibited poorer pathological features, including positive lymph node involvement $(\mathrm{P}=0.003)$ and positive lymphovascular invasion $(\mathrm{P}=0.010$; Table IV). Additionally, adjuvant chemotherapy did not significantly improve OS $(\mathrm{P}=0.167)$ or CSS rates $(\mathrm{P}=0.143$; Fig. 5) in patients with advanced $\mathrm{pT}$ stage $(\geq \mathrm{T} 3)$. 
Table IV. Clinicopathological characteristics of the 54 patients with advanced pathological T stage ( $\geq \mathrm{T} 3)$ according to administration of adjuvant chemotherapy.

\begin{tabular}{|c|c|c|c|}
\hline \multirow[b]{2}{*}{ Characteristics } & \multicolumn{2}{|c|}{ Adjuvant chemotherapy } & \multirow[b]{2}{*}{ P-value } \\
\hline & Yes $(n=29)$ & No $(n=25)$ & \\
\hline Median age, years (IQR) & $70(64-75)$ & $74(67-78)$ & 0.099 \\
\hline \multicolumn{4}{|l|}{ Sex, n $(\%)$} \\
\hline Male & $16(55.2)$ & $17(68)$ & \multirow[t]{2}{*}{0.407} \\
\hline Female & $13(44.8)$ & $8(32)$ & \\
\hline \multicolumn{4}{|l|}{ Tumor location, n (\%) } \\
\hline Renal pelvis & $18(62.1)$ & $19(76)$ & \multirow[t]{2}{*}{0.380} \\
\hline Ureter & $11(37.9)$ & $6(24)$ & \\
\hline \multicolumn{4}{|l|}{ Hydronephrosis, n (\%) } \\
\hline Yes & $20(69)$ & $12(48)$ & \multirow[t]{2}{*}{0.167} \\
\hline No & $9(31)$ & $13(52)$ & \\
\hline Median tumor size, $\mathrm{cm}$ (IQR) & $3.2(2.3-4.4)$ & $3.5(2.0-4.1)$ & 0.748 \\
\hline \multicolumn{4}{|l|}{ pT stage, n (\%) (16) } \\
\hline pT3 & $24(82.8)$ & $24(96)$ & \multirow[t]{2}{*}{0.200} \\
\hline pT4 & $5(17.2)$ & $1(4)$ & \\
\hline \multicolumn{4}{|l|}{ Lymph node involvement, n (\%) } \\
\hline $\mathrm{pNx} / \mathrm{NO}$ & $15(51.7)$ & $22(88)$ & \multirow[t]{2}{*}{$0.003^{t}$} \\
\hline $\mathrm{pN} 1$ & $14(48.3)$ & $3(12)$ & \\
\hline \multicolumn{4}{|l|}{ Tumor grade, n (\%) } \\
\hline $\mathrm{G} 1 / \mathrm{G} 2$ & $10(34.5)$ & $14(56)$ & \multirow[t]{2}{*}{0.170} \\
\hline G3 & $19(65.5)$ & $11(44)$ & \\
\hline \multicolumn{4}{|l|}{ Lymphovascular invasion, n (\%) } \\
\hline Yes & $24(82.8)$ & $12(50)$ & \multirow[t]{2}{*}{$0.010^{\mathrm{t}}$} \\
\hline No & $5(17.2)$ & $12(50)$ & \\
\hline \multicolumn{4}{|l|}{ Surgical margins, n (\%) } \\
\hline Positive & $8(27.6)$ & $3(12)$ & \multirow[t]{2}{*}{0.191} \\
\hline Negative & $21(72.4)$ & $22(88)$ & \\
\hline
\end{tabular}

${ }^{\text {aS }}$ Statistical analysis was performed by Mann-Whitney U test for continuous variables or $\chi 2$ test for categorical variables. ${ }^{\mathrm{b}}$ Statistically significant $(\mathrm{P}<0.05)$. G, grade; IQR, interquartile range; $\mathrm{pN}$, pathological $\mathrm{N} ; \mathrm{pT}$, pathological $\mathrm{T}$.

\section{Discussion}

Compared with bladder cancer, there is insufficient evidence regarding the clinical significance of neoadjuvant chemotherapy in patients with UTUC $(6,19)$. Additionally, accurate preoperative staging by imaging examination remains challenging. Currently, numerous institutions perform nephroureterectomy without neoadjuvant chemotherapy immediately following a diagnosis of UTUC without metastasis (20). Consequently, $\sim 40.0 \%$ of patients are diagnosed with advanced pT stage ( $\geq \mathrm{T} 3$ ) and are required to consider adjuvant chemotherapy (5). Therefore, it is important to investigate postoperative prognostic markers, as well as preoperative prognostic markers in patients with UTUC.

Previously, a number of studies reported that a high NLR is associated with poor survival in patients with numerous types of malignancy, including lung, renal, gastric, hepatic and colorectal cancer $(9,10)$. With respect to UTUC, a high NLR is associated with a reduced prognosis, compared with a low NLR $(14,15,18)$. Numerous studies demonstrated an association between preoperative NLR and survival $(14,15,18)$. By contrast, the present study aimed to investigate whether postoperative NLR is an effective prognostic marker for patients with clinically localized UTUC who have undergone surgical treatment.

The postoperative NLR has been investigated as a beneficial prognostic marker not only in urothelial carcinoma $(21,22)$, but also in lung and gastric cancer $(23,24)$. Kang et al (21), reported that early postoperative NLR is significantly associated with poor OS and CSS rates in patients who have undergone a radical cystectomy. Morizawa et al (22), demonstrated that a high postoperative NLR is associated with tumor recurrence even before a mass is detected by imaging examination in patients who have undergone radical cystectomy. The present results revealed that a high early postoperative NLR was significantly associated with a poor prognosis in patients with clinically localized UTUC. Among those with advanced pT stage ( $\geq \mathrm{T} 3$ ), patients with a high postoperative NLR exhibited 

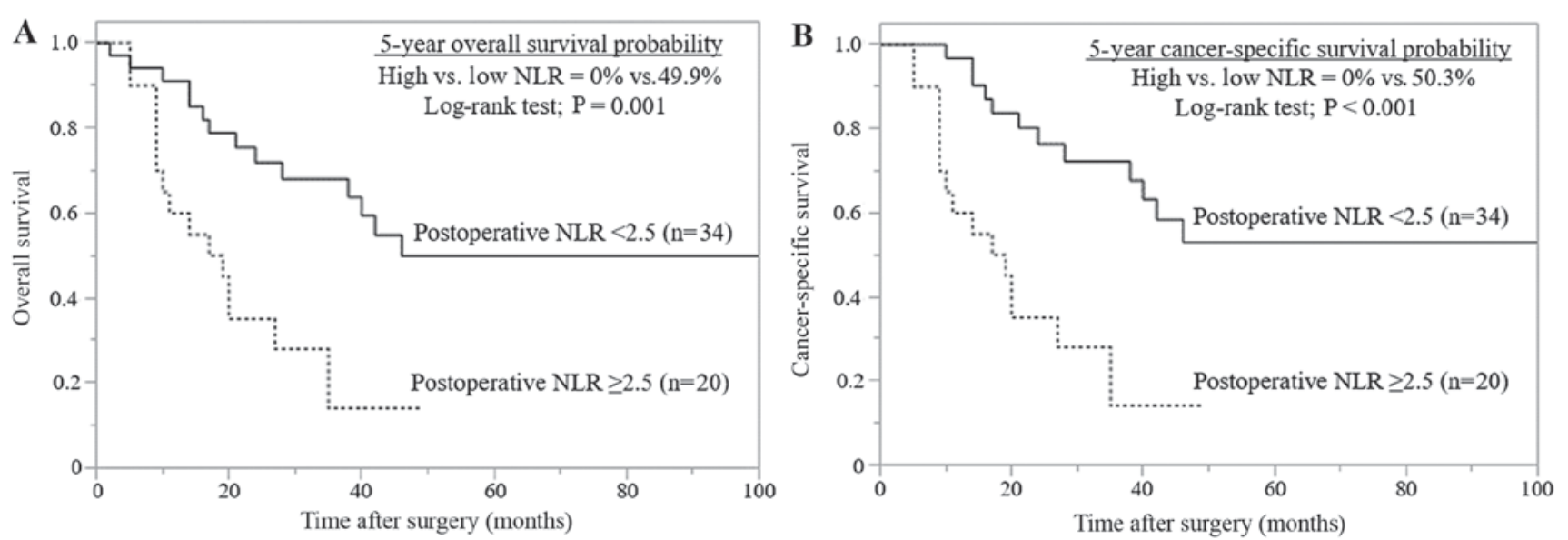

Figure 4. Survival analysis according to postoperative NLR for the 54 patients with advanced pathological T stage ( $\geq$ T3). Kaplan-Meier curves for (A) OS and (B) CSS. NLR, neutrophil-to-lymphocyte ratio; OS, overall survival; CSS, cancer-specific survival.
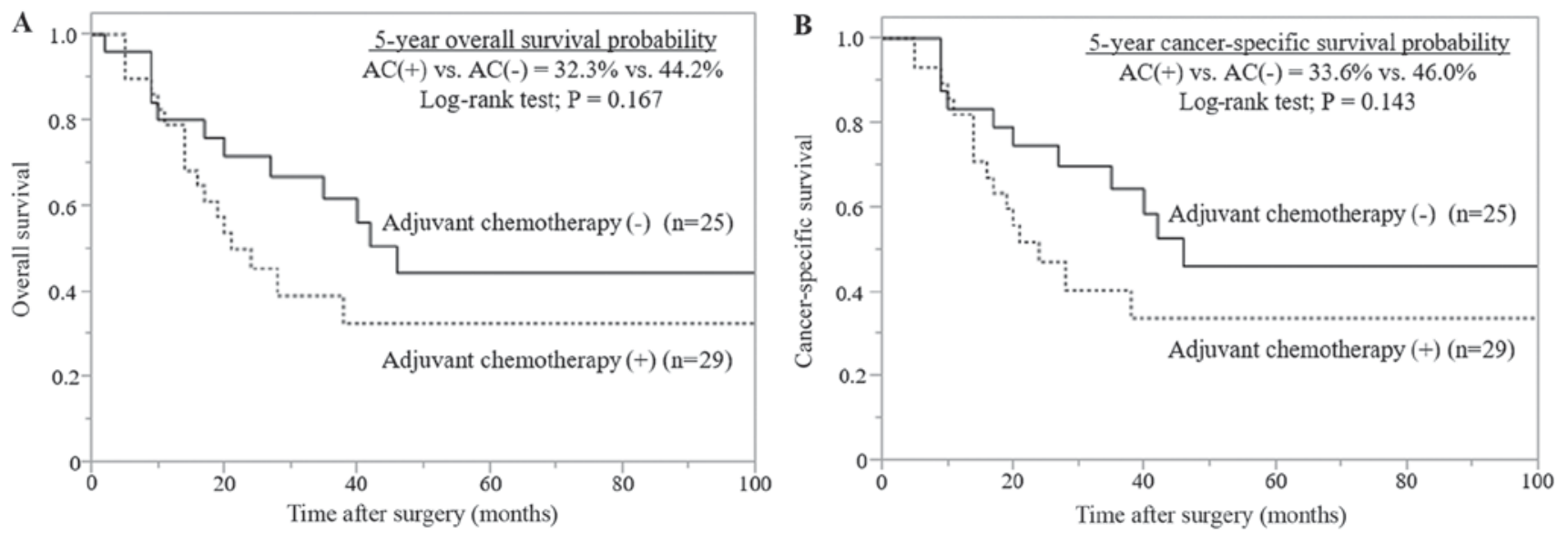

Figure 5. Survival curves of the 54 patients with advanced pathological T stage ( $\geq \mathrm{T} 3$ ) UTUC according to the presence or absence of AC. (A) OS and (B) CSS AC, adjuvant chemotherapy; OS, overall survival; CSS, cancer-specific survival.

a significantly reduced prognosis, compared with those with a low postoperative NLR. Furthermore, a high preoperative NLR was identified as a significant prognostic marker in the present study. This may be because a high postoperative NLR reflects only the potentially residual cancer, including micrometastases, while preoperative NLR reflects the primary tumor and the potential micrometastatic lesion. If the postoperative NLR reflects micrometastases, it appears to be a significant prognostic marker in patients with surgical treatment.

Recently, a phase III randomized trial of perioperative chemotherapy versus surveillance in UTUC (POUT trial, NCT01993979) reported that adjuvant chemotherapy improved the disease-free survival rate for patients with histologically confirmed pT2-T4 N0-3 M0 UTUC (25). However, it could not be accurately determined whether the primary lesion was localized, as the current imaging modalities are not able to detect disseminated micrometastases. In this sense, the present study provided reasonable evidence that postoperative NLR may be a beneficial marker to select patients who require adjuvant therapy.

To the best of our knowledge, the mechanisms underlying the association between a high NLR and poor survival in patients with cancer remain unclear. A study by Mantovani et al (26), supported the involvement of systemic inflammation in cancer development and progression. Features of cancer-associated inflammation involve the secretion of inflammatory molecules, including cytokines and prostaglandins, and the recruitment of inflammatory cells into the tumor tissue (26). The cytokines activate the same key transcription factors in inflammatory cells, stromal cells and tumor cells, resulting in more inflammatory mediators being produced and a cancer-associated inflammatory microenvironment being generated (26). Inflammatory cytokines and chemokines can be produced by the tumor and associated host cells, including the leukocytes neutrophils and monocytes, and contribute to malignant progression $(26,27)$. Lymphocytes serve a fundamental role in antitumor immunity (28). It has been reported that the increasing infiltration of tumors by lymphocytes is associated with an improved response to cytotoxic treatment and prognosis in patients with cancer (28). Lymphopenia has been demonstrated to be an independent predictor of poor survival in pancreatic and renal cell cancer $(29,30)$. Furthermore, the neutrophil response is associated with the cancer progression process and lymphocytes serve an important role in immune surveillance of cancer (26-30). Therefore, the NLR represents both groups by a simple measurement and could be an indicator of homeostasis between cancer progression and antitumor activity. 
There are several limitations of the present study. Firstly, the present study was a retrospective non-randomized analysis. Therefore, it may not be possible to completely avoid selection and information bias. Secondly, only a relatively small number of patients were included in the study. A limited in the number of patients with UTUC were included in the study, as UTUC is uncommon, accounting for 5-10\% of all urothelial cancer cases $(2,3)$. Thirdly, the present study had a relatively short median follow-up duration of 40.5 months and the time span was relatively large. Although the timespan of the observation period was relatively large, the data analysis was appropriately conducted and the timespan did not affect the analysis result. Fourthly, adjuvant chemotherapy did not exhibit a significant benefit in terms of OS or CSS in the present study. This may be due to the small population size, regimen variability, patient selection bias and a small number of chemotherapy cycles. In the present study, the NLR was measured once preoperatively and once postoperatively. There is a tendency for a high NLR to also reflect infection and stress, in addition to cancer (31). Therefore, it is necessary to consider the timing of measuring the postoperative NLR.

In conclusion, a high postoperative NLR is associated with a poor prognosis in patients with UTUC. The postoperative NLR may be a more accurate prognostic marker, compared with the preoperative NLR, in patients with UTUC. The present study indicates that the postoperative NLR may be a potential prognostic marker, in addition to pathological features. Identifying patients with a increased chance of survival may assist with the development of adjuvant therapy for specific subgroups of patients to improve survival or establish individualized follow-up protocols. However, further external validation and a prospective multicenter trial is necessary to confirm the prognostic significance of the postoperative NLR in patients with UTUC.

\section{Acknowledgements}

Not applicable.

\section{Funding}

The present study was supported by the Japan Society for the Promotion of Science (KAKENHI grant no. JP18K09182).

\section{Availability of data and materials}

The datasets used and/or analyzed during the present study are available from the corresponding author on reasonable request.

\section{Authors' contributions}

KN, SS and TI designed the study and revised the manuscript. $\mathrm{KU}, \mathrm{MN}$ and $\mathrm{MM}$ contributed to the collection, analysis and interpretation of the data.

\section{Ethics approval and consent to participate}

The present study was approved by the Research Ethics Committee of Kurume University (Fukuoka, Japan; approval no. 17303). The requirement for informed consent was waived due to the retrospective nature of the study. The research content was available publicly on the website of the Research Ethics Committee of Kurume University, which ensured opportunities for participants to opt out of the research.

\section{Patient consent for publication}

Not applicable.

\section{Competing interests}

The authors declare that they have no competing interests.

\section{References}

1. Oya M and Kikuchi E; Committee for Establishment of Clinical Practice Guideline for Management of Upper Tract Urothelial Carcinoma; Japanese Urological Association: Evidenced-based clinical practice guideline for upper tract urothelial carcinoma (summary--Japanese Urological Association, 2014 edition). Int J Urol 22: 3-13, 2015.

2. Siegel R, Naishadham D and Jemal A: Cancer statistics, 2012. CA Cancer J Clin 62: 10-29, 2012.

3. Rouprêt M, Babjuk M, Compérat E, Zigeuner R, Sylvester RJ, Burger M, Cowan NC, Böhle A, Van Rhijn BWG, Kaasinen E, et al: European Association of Urology Guidelines on Upper Urinary Tract Urothelial Cell Carcinoma: 2015 Update. Eur Urol 68: 868-879, 2015.

4. Ministry of Health, Labour and Welfare: Vital Statistics of Japan (Volume 1 3), General Mortality. https://www.mhlw.go.jp/ english/database/db-hw/vs01.html. Accessed September 7, 2018.

5. Margulis V, Shariat SF, Matin SF, Kamat AM, Zigeuner R, Kikuchi E, Lotan Y, Weizer A, Raman JD and Wood CG; Upper tract urothelial carcinoma collaboration the upper tract urothelial carcinoma collaboration: outcomes of radical nephroureterectomy: A series from the upper tract urothelial carcinoma collaboration. Cancer 115: 1224-1233, 2009.

6. Witjes JA, Compérat E, Cowan NC, De Santis M, Gakis G, Lebret T, Ribal MJ, Van der Heijden AG and Sherif A; European Association of Urology: EAU guidelines on muscle-invasive and metastatic bladder cancer: Summary of the 2013 guidelines. Eur Urol 65: 778-792, 2014.

7. Rink M, Sjoberg D, Comploj E, Margulis V, Xylinas E, Lee RK, Hansen J, Cha EK, Raman JD, Remzi M, et al: Risk of cancer-specific mortality following recurrence after radical nephroureterectomy. Ann Surg Oncol 19: 4337-4344, 2012.

8. Leow JJ, Orsola A, Chang SL and Bellmunt J: A contemporary review of management and prognostic factors of upper tract urothelial carcinoma. Cancer Treat Rev 41: 310-319, 2015.

9. Guthrie GJ, Charles KA, Roxburgh CS, Horgan PG, McMillan DC and Clarke SJ: The systemic inflammation-based neutrophil-lymphocyte ratio: Experience in patients with cancer. Crit Rev Oncol Hematol 88: 218-230, 2013.

10. Templeton AJ, McNamara MG, Šeruga B, Vera-Badillo FE, Aneja P, Ocaña A, Leibowitz-Amit R, Sonpavde G, Knox JJ, Tran B, et al: Prognostic role of neutrophil-to-lymphocyte ratio in solid tumors: A systematic review and meta-analysis. J Natl Cancer Inst 106: dju124, 2014.

11. Viers BR, Boorjian SA, Frank I, Tarrell RF, Thapa P, Karnes RJ, Thompson RH and Tollefson MK: Pretreatment neutrophil-to-lymphocyte ratio is associated with advanced pathologic tumor stage and increased cancer-specific mortality among patients with urothelial carcinoma of the bladder undergoing radical cystectomy. Eur Urol 66: 1157-1164, 2014.

12. Gondo T, Nakashima J, Ohno Y, Choichiro O, Horiguchi Y, Namiki K, Yoshioka K, Ohori M, Hatano T and Tachibana M: Prognostic value of neutrophil-to-lymphocyte ratio and establishment of novel preoperative risk stratification model in bladder cancer patients treated with radical cystectomy. Urology 79: 1085-1091, 2012.

13. Krane LS, Richards KA, Kader AK, Davis R, Balaji KC and Hemal AK: Preoperative neutrophil/lymphocyte ratio predicts overall survival and extravesical disease in patients undergoing radical cystectomy. J Endourol 27: 1046-1050, 2013. 
14. Tanaka N, Kikuchi E, Kanao K, Matsumoto K, Shirotake S, Miyazaki Y, Kobayashi H, Kaneko G, Hagiwara M, Ide H, et al: A multi-institutional validation of the prognostic value of the neutrophil-to-lymphocyte ratio for upper tract urothelial carcinoma treated with radical nephroureterectomy. Ann Surg Oncol 21: 4041-4048, 2014

15. Dalpiaz O, Ehrlich GC, Mannweiler S, Hernández JM, Gerger A, Stojakovic T, Pummer K, Zigeuner R, Pichler M and Hutterer GC: Validation of pretreatment neutrophil-lymphocyte ratio as a prognostic factor in a European cohort of patients with upper tract urothelial carcinoma. BJU Int 114: 334-339, 2014

16. Sobin LH, Gospodarowicz MK and Wittekind C (eds): UICC TNM Classification of Malignant Tumours. 7th edition. Wiley-Blackwell, New Jersey, NY, 2009.

17. Mostofi FK, Davis CJ and Sesterhenn IA: World Health Organization International Histological Classification of Tumours. Histological typing of urinary bladder tumours. 2nd edition. Springer-Verlag Berlin, Heidelberg, 1999.

18. Azuma T, Matayoshi Y, Odani K, Sato Y, Sato Y, Nagase Y and Oshi M: Preoperative neutrophil-lymphocyte ratio as an independent prognostic marker for patients with upper urinary tract urothelial carcinoma. Clin Genitourin Cancer 11: 337-341, 2013.

19. Advanced Bladder Cancer (ABC) Meta-analysis Collaboration: Neoadjuvant chemotherapy in invasive bladder cancer: Update of a systematic review and meta-analysis of individual patient data advanced bladder cancer (ABC) meta-analysis collaboration. Eur Urol 48: 202-205, discussion 205-206, 2005.

20. Fritz GA, Schoellnast H, Deutschmann HA, Quehenberger F and Tillich M: Multiphasic multidetector-row CT (MDCT) in detection and staging of transitional cell carcinomas of the upper urinary tract. Eur Radiol 16: 1244-1252, 2006.

21. Kang M, Jeong CW, Kwak C, Kim HH and Ku JH: The prognostic significance of the early postoperative neutrophil-to-lymphocyte ratio in patients with urothelial carcinoma of the bladder undergoing radical cystectomy. Ann Surg Oncol 23: 335-342, 2016

22. Morizawa Y, Miyake M, Shimada K, Hori S, Tatsumi Y, Nakai $\mathrm{Y}$, Anai S, Tanaka N, Konishi $\mathrm{N}$ and Fujimoto $\mathrm{K}$ : Neutrophil-to-lymphocyte ratio as a detection marker of tumor recurrence in patients with muscle-invasive bladder cancer after radical cystectomy. Urol Oncol 34: 257 e211-257, 2016. doi. org/10.1016/j.urolonc.2016.02.012.
23. Tanaka H, Tamura T, Toyokawa T, Muguruma K, Miki Y, Kubo N, Sakurai K, Hirakawa K and Ohira M: Clinical relevance of postoperative neutrophil-lymphocyte ratio (NLR) to recurrence after adjuvant chemotherapy of S-1 for gastric cancer. Anticancer Res 38: 3745-3751, 2018.

24. Jin F, Han A, Shi F, Kong L and Yu J: The postoperative neutrophil-to-lymphocyte ratio and changes in this ratio predict survival after the complete resection of stage I non-small cell lung cancer. OncoTargets Ther 9: 6529-6537, 2016.

25. Birtle AJ, Chester JD, Jones RJ, Johnson M, Hill M, Bryan RT, Catto J, Donovan J, French A, Harris C, et al: Results of POUT: A phase III randomised trial of perioperative chemotherapy versus surveillance in upper tract urothelial cancer (UTUC). J Clin Oncol 36 (6_suppl): 407-407, 2018.

26. Mantovani A, Allavena P, Sica A and Balkwill F: Cancer-related inflammation. Nature 454: 436-444, 2008.

27. Balkwill $\mathrm{F}$ and Mantovani A: Inflammation and cancer: Back to Virchow? Lancet 357: 539-545, 2001.

28. Gooden MJ, de Bock GH, Leffers N, Daemen T and Nijman HW: The prognostic influence of tumour-infiltrating lymphocytes in cancer: A systematic review with meta-analysis. Br J Cancer 105: 93-103, 2011.

29. Fogar P, Sperti C, Basso D, Sanzari MC, Greco E, Davoli C, Navaglia F, Zambon CF, Pasquali C, Venza E, et al: Decreased total lymphocyte counts in pancreatic cancer: An index of adverse outcome. Pancreas 32: 22-28, 2006.

30. Saroha S, Uzzo RG, Plimack ER, Ruth K and Al-Saleem T: Lymphopenia is an independent predictor of inferior outcome in clear cell renal carcinoma. J Urol 189: 454-461, 2013.

31. Glaser R and Kiecolt-Glaser JK: Stress-induced immune dysfunction: Implications for health. Nat Rev Immunol 5: 243-251, 2005. 